\title{
Glycosylation of Zika Virus Is Important in Host-Virus Interaction and Pathogenesis
}

2 Nanda Kishore Routhu ${ }^{1}$, Sylvain D. Lehoux ${ }^{4,5}$, Emily A. Rouse ${ }^{5}$, Mehdi R. M. Bidokhti ${ }^{1}$, Leila 3 B. Giron ${ }^{6}$, Alit Anzurez ${ }^{6}$, St Patrick Reid ${ }^{7}$, Mohamed Abdel-Mohsen ${ }^{6}$, Richard D. Cummings ${ }^{4}$, 4 Siddappa N. Byrareddy ${ }^{1,2,3 *}$

$6 \quad{ }^{1}$ Department of Pharmacology and Experimental Neuroscience, University of Nebraska Medical

7 Center, Omaha, NE 68198-5800, USA;

$8{ }^{2}$ Department of Genetics, Cell Biology and Anatomy, University of Nebraska Medical Center, 9 Omaha, NE 68198-5805, USA;

$10{ }^{3}$ Department of Biochemistry and Molecular Biology, University of Nebraska Medical Center, 11 Omaha, NE 68198-5805, USA;

$12{ }^{4}$ Beth Israel Deaconess Medical Center, National Center for Functional Glycomics, Boston, MA, 13 USA;

$14{ }^{5}$ Beth Israel Deaconess Medical Center Glycomics Core, Boston, MA, USA;

$15{ }^{6}$ The Wistar Institute, Philadelphia, PA, USA;

$16{ }^{7}$ Department of Pathology and Microbiology, University of Nebraska Medical Center, Omaha, 17 NE, USA.

$21{ }^{*}$ Corresponding Author:

22 Siddappa Byrareddy, Ph.D.

23 Department of Pharmacology and Experimental Neuroscience

24 University of Nebraska Medical Center

25985800 Nebraska Medical Center

26 Omaha, NE 68198-5800 USA

27 Tel: (402) 559-5416

28 Fax: (402) 559-3744

29 Email: sid.byrareddy@unmc.edu

30 


\section{Abstract}

33 Zika virus (ZIKV) is a global public health issue due to its association with severe

34 developmental disorders in infants and neurological disorders in adults. Because ZIKV uses

35 glycosylation of its envelope (E) protein to interact with host cell receptors to facilitate entry,

36 these interactions could also be important for designing therapeutics and vaccines. Due to a lack

37 of information about Asn-linked (N-glycans) on ZIKV E, we analyzed ZIKV E of various strains

38 derived from different cells. ZIKV E proteins are extensively modified with oligomannose-,

39 hybrid- and complex-N-glycans of a highly heterogeneous nature. Host cell-surface glycans

40 correlated strongly with the glycomic features of ZIKV E. Mechanistically, we discovered that

41 ZIKV N-glycans are important in viral pathogenesis, as mannose-specific C-type lectins DC-

42 SIGN and L-SIGN mediate cell entry of ZIKV. Our findings represent the first detailed mapping

43 of N-glycans on ZIKV E of various strains and their functional significance. 
Zika virus (ZIKV) is mainly transmitted to humans via infected mosquitoes, though other

47 transmission routes, such as through placenta and sexual intercourse can also occur ${ }^{1,2}$. Recently, the considerable increase in ZIKV infection rates has raised urgent global urgent concerns, especially after the outbreaks in Yap Islands $(2007)^{3,4}$, French Polynesia (2013) ${ }^{5,6}$, Easter Island

$50(2014)^{7}$, and Brazil (2015) ${ }^{8,9}$. These ZIKV outbreaks were associated with a sharp increase in cases of Guillain Barre Syndrome (GBS), an autoimmune disease featured by weakening and

52 paralysis of the limbs and face. In 2015, Zika spread to South and Central America, infecting thousands of people in Brazil and Colombia, where it was associated with an increase in GBS rates as well as a significant increase in severe fetal abnormalities including spontaneous abortion, stillbirth, hydrocephaly, microcephaly, hydranencephaly/hydrops fetalis, and placental insufficiency ${ }^{10-14}$. ZIKV is a small, enveloped positive-strand RNA virus ${ }^{15}$. The genomic RNA

57 of this mosquito-borne flavivirus contains a single open reading frame (ORF) that encodes a 58 polyprotein ${ }^{16,17}$. This polyprotein undergoes co- and post-translational processing to produce 59 three structural proteins, which are capsid (C), pre-membrane (prM) and envelope (E), in addition to seven non-structural proteins (NS1, NS2A, NS2B, NS3, NS4A, NS4B, and NS5) ${ }^{17,18}$.

61 Of the three structural proteins, E is the major surface glycoprotein, containing three domains (I,

62 II, III) and two transmembrane helices ${ }^{17-19}$. The majority of flaviviruses E proteins are posttranslationally modified by N-glycans at amino acid 153/154 within a highly conserved 64 glycosylation motif of $\mathrm{N}-\mathrm{X}-\mathrm{T} / \mathrm{S}$ at positions $154-156$ (where $\mathrm{X}$ is any amino acid except proline)

$65{ }^{16}$. This is a primary target of neutralizing antibodies and is required for virus entry ${ }^{20}$.

66 Previously, it was demonstrated that the N-glycosylation sites on the E protein of flaviviruses are

67 highly conserved (Supplementary Fig. 1), playing a vital role in both infectivity and assembly 
69 secretion of E ectodomain from mammalian cells ${ }^{24}$. Furthermore, studies in mice have shown

70 that ZIKVs lacking the N-glycans of the ZIKV E were severely compromised for their ability to

71 cause mortality and neuroinvasion, suggesting a vital role for these glycans of the E protein in

72 ZIKV pathogenesis ${ }^{22,25}$. In this study, we utilized mass spectrometry (MS) and a lectin

73 microarray to analyze the structures and composition of glycans on ZIKV E from different

74 strains and on the surface of the virus-producing cells, as well as to explore mosquito-human

75 mode of ZIKV transmission and its association to neurological disorders. Importantly, our

76 functional studies show that these N-glycans of the ZIKV envelope glycoprotein are important in

77 viral infection of cells. Our findings have implications on understanding the roles for these

78 glycan patterns on ZIKV E in virus-host interaction and pathogenesis. 


\section{Results}

\section{Glycoprotein E localizes subcellularly in ZIKV-infected cells}

In order to confirm whether ZIKV E localized within the endoplasmic reticulum (ER), mammalian cells (Vero and SNB19 cells) and an insect cell line (C6/36 cells) were infected with the following ZIKV strains 1) Asian strains (PRVABC59 and FLR isolates), 2) African strains (MR766 and IbH isolates) and 3) a Brazilian isolate from 2016 (SJRP) for 24 hours. As shown in Fig. 1, the patterns of fluorescence demonstrated that ZIKV E localizes to the periphery of cell nucleus, consistent with localization in the ER. The cytoplasmic area of Vero cells and SNB19 cells that were infected with PRVABC59 and MR766 displayed similar patterns of perinuclear and nuclear immunofluorescence of ZIKV E. However, C6/36 cells are smaller in size compared to other cell lines and displayed reduced cytoplasmic area compared to uninfected cells. The cells that were infected with either the MR766 or IbH strain exhibited particularly reduced cytoplasmic area. These data suggest that the ZIKV E localizes predominantly to ER.

\section{MS analysis of ZIKV E derived from different cell lines showed various profiles of $\mathrm{N}$ -} glycan pattern

Following the production and purification of the different ZIKV strains in various cell lines, the virions were pelleted, lysed, resolved in 12\% bis-tris protein gels, and stained with Coomassie Blue. The bands corresponding to the ZIKV E were excised for N-glycan MS profiling (Supplementary Fig. 3). The relative abundances of the N-glycans released by PNGase F, and identified for each of the five ZIKV E proteins produced in each of the six different cell lines, are presented as a heatmap in Fig. 2; the predicted glycan structures with the corresponding sizes are presented in Supplementary Table 1. Although distinct N-glycosylation patterns emerged, the 
two ZIKV E proteins from African lineage strains, MR766 and IbH, displayed similar N-glycan profiles across the six cell lines that were tested (Supplementary Fig. 4a, b). When produced in C6/36, Vero, THP-1, and SNB19 cells, these N-glycan profiles were heavily dominated by sialylated glycans. For these four cell lines, bi- and tri-sialylated tri-antennary N-glycans (3241.8 $\mathrm{m} / \mathrm{z}$ and $3603.0 \mathrm{~m} / \mathrm{z}$ ) were the most abundant found on ZIKV E, followed by bi-sialylated biantennary $(2792.5 \mathrm{~m} / \mathrm{z})$ and tetra-sialylated tri-antennary $(3964.2 \mathrm{~m} / \mathrm{z}) \quad \mathrm{N}$-glycans (Supplementary Fig. 4a, b). ZIKV E produced in SNB19 cells was also found to be decorated with Man5 $(1579.9 \mathrm{~m} / \mathrm{z})$, agalactosylated bisected $(1907.1 \mathrm{~m} / \mathrm{z})$, core-fucosylated non-sialylated bi-antennary $(2244.3 \mathrm{~m} / \mathrm{z})$, and core-fucosylated mono-sialylated bi-antennary $(2605.5 \mathrm{~m} / \mathrm{z}) \mathrm{N}$ glycans (Supplementary Fig. 4a, b). When expressed in the LLC-MK2 and JEG-9 cell lines, the N-glycan profiles of MR766 and IbH strains of E were markedly different. When produced in LLC-MK2 cells, bi-sialylated bi-antennary $(2792.5 \mathrm{~m} / \mathrm{z})$ and core-fucosylated agalactosylated (1836.0 m/z) N-glycans were by far the two most abundant glycan species (Supplementary Fig. 4a, b). More complex N-glycans, with three or more sialic acids and additional lactosamine repeats, were not identified in ZIKV E expressed in LLC-MK2 cells. Finally, the ZIKV E produced in JEG-3 cells revealed a different N-glycosylation pattern between MR766 and IbH strains. While the di- and tri-sialylated N-glycans $(2792.5,3241.8$, and $3603.0 \mathrm{~m} / \mathrm{z})$ were the most abundant for the MR766 ZIKV-E produced in JEG-3, the variety of N-glycans identified in JEG-3 cells was greater, notably including Man ${ }_{5} \operatorname{GlcNAc}_{2}(1579.9 \mathrm{~m} / \mathrm{z}), \mathrm{Man}_{6} \mathrm{GlcNAc}_{2}(1784.0$ $\mathrm{m} / \mathrm{z}), \operatorname{Man}_{7} \mathrm{GlcNAc}_{2}(1988.1 \mathrm{~m} / \mathrm{z}), \mathrm{Man}_{8} \mathrm{GlcNAc}_{2}(2192.2 \mathrm{~m} / \mathrm{z})$, asialo bi-antennary (2070.2 $\mathrm{m} / \mathrm{z}$ ), and core fucosylated asialo-bi-antennary $(2244.3 \mathrm{~m} / \mathrm{z}$ ) N-glycans (Supplementary Fig. 4a). In contrast, the IbH strain produced in the JEG-3 cells displayed a more restricted N-glycan profile, with the notable absence of sialylated N-glycans and with the core fucosylated asialo-bi- 
125 126

127

128

129

130

131

132

133

134

135

136

137

138

139

140

141

142

143

144

145

146

147

antennary $(2244.3 \mathrm{~m} / \mathrm{z}) \mathrm{N}$-glycan being the most complex N-glycan identified (Supplementary Fig. 4b).

Similar to African lineage ZIKV, the N-glycosylation profiles from the Asian lineage viruses PRVABC59 and FLR varied across cell lines, yet were nearly identical within any given cell line (Supplementary Fig. 4c, d). Additionally, the E protein of PRVABC59 and FLR strains expressed in C6/36, Vero, THP-1, and SNB19 cell lines were primarily decorated with trisialylated tri-antennary N-glycans $(3603.0 \mathrm{~m} / \mathrm{z})$ and — with a lesser abundance- di-sialylated biand tri-antennary $(2792.5$ and $3241.8 \mathrm{~m} / \mathrm{z})$ and tetra-sialylated tri-antennary $(3964.2 \mathrm{~m} / \mathrm{z}) \mathrm{N}$ glycans (Supplementary Fig. 4c, d). Minor N-glycans including Man ${ }_{5} \operatorname{GlcNAc}_{2}(1579.9 \mathrm{~m} / \mathrm{z}$ ), monosialylated bi-antennary $(2431.4 \mathrm{~m} / \mathrm{z})$, core-fucosylated mono-galactosylated bi-antennary (2040.2 m/z), core-fucosylated asialo bi-antennary $(2244.3 \mathrm{~m} / \mathrm{z})$, and core-fucosylated monosialylated bi-antennary (2605.5 m/z) N-glycans were also identified (Supplementary Fig. 4c, d). Similar to the African lineage, the E proteins of Asian lineage revealed a more restricted Nglycan profile when expressed in LLC-MK2 cells. The di-sialylated bi-antennary $(2792.5 \mathrm{~m} / \mathrm{z})$ $\mathrm{N}$-glycan was identified as the most abundant $\mathrm{N}$-glycan, with very low to no expression of more complex N-glycans. The core-fucosylated agalactosylated $(1836.0 \mathrm{~m} / \mathrm{z})$ and mono-sialylated biantennary $(2431.4 \mathrm{~m} / \mathrm{z})$ were, respectively, the second and third most relatively abundant $\mathrm{N}$ glycans reported (Supplementary Fig. 4c, d). Lastly, the N-glycan profiles of the PRVABC59 and FLR strains of E expressed in JEG-3 cells showed a lower abundance of highly sialylated Nglycans such as the tri- and tetra-sialylated tri-antennary N-glycans (3603.0 and $3964.2 \mathrm{~m} / \mathrm{z}$ ) compared to the virus produced in the C6/36, Vero, THP-1, and SNB19 cells. By contrast, the E of the PRVABC59 strain showed an increased abundance of both mono-sialylated N-glycans (2431.4 and $2880.6 \mathrm{~m} / \mathrm{z}$ ) and non-sialylated N-glycans $(2040.2,2070.2$ and $2244.3 \mathrm{~m} / \mathrm{z}$ ). 
Unlike the African and Asian lineage strains, the N-glycans found on the E protein of the Brazilian isolate SJRP were almost exclusively non-sialylated when produced in C6/36 and Vero cells. In both of these cell lines, the major N-glycans found were $\mathrm{Man}_{5} \mathrm{GlcNAc}_{2}(1579.9 \mathrm{~m} / \mathrm{z})$, asialo-bi-antennary $(2070.2 \mathrm{~m} / \mathrm{z})$, and core fucosylated asialo-bi-antennary $(2244.3 \mathrm{~m} / \mathrm{z}) \mathrm{N}$ glycans (Supplementary Fig. 4e). When produced in LLC-MK2 cells, however, the N-glycan profile of the E protein from the SJRP strain was comparable to the profiles found for the four other strains, with the di-sialylated bi-antennary $(2792.6 \mathrm{~m} / \mathrm{z})$ and the core-fucosylated agalactosylated $(1836.1 \mathrm{~m} / \mathrm{z}) \mathrm{N}$-glycans being the most abundant. Contrasting the other E proteins produced in C6/36, LLC-MK2 and Vero cells, Man5 $(1579.9 \mathrm{~m} / \mathrm{z})$ was found to be the most abundant N-glycan on the SJRP strain. However, the N-glycan profiles generated from the E protein of SJRP strain expressed in THP-1, SNB19, and JEG-3 cells revealed a high relative abundance of di-sialylated bi-antennary $(2792.5 \mathrm{~m} / \mathrm{z})$, tri-antennary $(3241.8 \mathrm{~m} / \mathrm{z})$, and trisialylated tri-antennary $(3603.0 \mathrm{~m} / \mathrm{z})$ with little to no expression of less complex non-sialylated N-glycans such as $\mathrm{Man}_{5} \mathrm{GlcNAc}_{2}$ (Supplementary Fig. 4e).

The major proportion ( $>$ 5\%) of N-glycan spectra at $3603.0 \mathrm{mz}, 3241.8 \mathrm{mz}, 2792.6 \mathrm{mz}, 3964.2$ $\mathrm{mz}, 1836.0 \mathrm{mz}$, and $2040.2 \mathrm{mz}$ were identified on ZIKV E produced in these cell lines. These heterogenous $\mathrm{N}$-glycan structures of the studied ZIKV E include high (oligo)-mannose; these and other N-glycans compositionally are predicted to include the residues $\mathrm{N}$-acetylgalactosamine (GalNAc), N-acetylglucosamine (GlcNAc), N-acetylneuraminic acid (NeuAc), galactose (Gal), glucose (Glc), fucose (Fuc), and sialic acid. In addition, we found many other glycan forms with high heterogeneity but smaller proportion $(<5 \%)$; these were not further characterized. These data are indicative of high heterogeneity of structure and composition of N-glycans on ZIKV E proteins (Supplementary Table 2). 


\section{Cell-surface glycosylation strongly correlates with glycomic features of ZIKV E protein}

172 In order to identify the general patterns of endogenous glycans in ZIKV-producing cell surface

173 endogenous glycoproteins, a lectin array was used. This approach relies on specificities of

174 immobilized lectins that recognize specific glycan features on cell surface glycoproteins,

175 resulting in their binding to the array. These data revealed that different cell lines harbor

176 differential cell-surface glycosylation (Fig. 3) that might impact the glycosylation of the virus

177 produced in these cells. In general, the expression of several cell-surface glycan structures

178 correlated with viral N-glycan features (Supplementary Table 3). Of interest, and as shown in

179 Fig. 4, strong correlation between the presence of cell-surface Fuc, sialic acid, lactose, mannose,

180 GlcNAc, and the N-glycan features of E-protein. There was no statistically significant difference

181 with other proteins analyzed.

\section{C-type Lectins DC-SIGN and L-SIGN play a functional important role in ZIKV infection}

The C-type lectins such as DC-SIGN (dendritic cell-specific ICAM-grabbing non-integrin, where ICAM is an intercellular adhesion molecule) and L-SIGN (where L is liver or lymph node) have considerable binding affinity to viral mannose-rich glycan ${ }^{37,38}$. DC-SIGN is expressed on various cell types such as monocytes and dendritic cells (DCs), and L-SIGN is expressed on endothelial cells of liver and lymph nodes (L) and recognizes carbohydrate structures present on viral glycoproteins ${ }^{38}$. Thus, these lectins function as attachment factors for several enveloped viruses including human immunodeficiency virus-1 (HIV-1), Ebola (EBOV), Japanese encephalitis virus (JEV), and Dengue virus (DENV) ${ }^{39,40}$. Given that ZIKV envelope- 
194 the mouse embryonic fibroblast cells) expressing DC-SIGN (DC-SIGN/3T3 cells) and L-SIGN

195 (L-SIGN/3T3 cells) receptors, along with only 3T3/NIH cells as a control. Cells were infected 196 with ZIKV at a MOI of 1, and the percent infection was determined after 24 hours through the 197 use of an immunofluorescence analysis and a high-throughput Operetta imager. As presented in 198 Fig. 5, the percentage (\%) of infected cells increased in DC-SIGN and L-SIGN expressing cells 199 compared to control 3T3/NIH cells, indicating that DC- and L-SIGN expressing 3T3 cells are 200 susceptible to ZIKV infection (Fig. 5a). To further confirm the role of high-mannose type 201 glycans, we performed experiments in the presence of mannan and EDTA. EDTA blocks DC202 SIGN by extracting the bound calcium, while Mannan competes with insect-derived high203 mannose type glycans and both inactivate DC-SIGN ${ }^{41}$. Both mannan and EDTA inhibited ZIKV 204 replication efficiently (Fig. 5b, c). These results suggest that ZIKV infection efficiency is 205 dependent upon high-mannose type glycans, and the entry is mediated through DC-SIGN/LSIGN cell surface expression. Taken together, DC-SIGN/L-SIGN receptors are essential for optimizing the entry and infection of various strains of ZIKV.

\section{Discussion} towards therapeutic discovery and vaccine development. It is equally important to understand how glycosylation affects the biological properties and pathogenesis of the virus, and for

214 characterize the E protein N-glycans of ZIKV derived from insect cells and mammalian cells.

215 We showed for the first time that ZIKV E, which is required for host cell receptor binding, is 216 differentially glycosylated in a cell-type different fashion. The N-glycosylation pattern on ZIKV 
217 E is thought to be involved in viral infectivity, morphogenesis, protein folding, virus entry, and

218 tissue tropism among other functions. This has also been described for other viruses including 219 influenza virus ${ }^{43}$, HIV-1, West Nile Virus, JEV, hepatitis C virus (HCV), Nipah virus, EBOV, 220 and sever acute respiratory syndrome (SARS) coronavirus ${ }^{44}$. Analyses of the N-glycans on 221 ZIKVs grown in insect and various mammalian cells revealed a high degree of heterogeneity.

222 The variety of N-glycans found on ZIKV E ranged from high- (or oligo-) mannose, notably $223 \mathrm{Man}_{5} \mathrm{GlcNAc}_{2}$, to highly sialylated (up to four sialic acids) complex-type N-glycans and with 224 very few hybrid types. However, both African and Asian lineage strains revealed very similar N225 glycosylation patterns of ZIKV E. Di- and tri-sialylated bi- and tri-antennary were the most 226 relatively abundant $\mathrm{N}$-glycans found on ZIKV E when viruses were produced in C6/36 insect 227 cells and LLC-MK2, Vero, THP-1, SNB19, and JEG-3 mammalian cells. High-mannose, asialo228 and agalacto-N-glycans were also identified, though in lesser overall relative abundance. The 229 presence of high-mannose and complex-type N-glycans on mammalian cell-derived virus is not 230 limited to ZIKV as has been reported for DENV serotypes ${ }^{45,46}$, and is the most abundant type of 231 N-glycans found on HIV GP120 glycoprotein ${ }^{47}$. On the other hand, any ZIKV strain when 232 produced in various cell lines showed different N-glycan patterns. Indeed, from a cell line 233 perspective, LLC-MK2 cells produced the same unique N-glycosylation pattern for all five 234 ZIKV E, which were mostly decorated with bi-antennary di-sialylated and core-fucosylated 235 agalactosylated N-glycans (Fig. 2-3, Supplementary Fig. 4). Collectively, these findings 236 indicate that not only virus characteristics; e.g. strain or lineage, but also host cell physiology has 237 an impact on the glycosylation pattern of ZIKV E which may affect viral pathogenesis of ZIKV 238 strains. 
In the current study, we did not determine the location of the N-glycans on ZIKV E protein. We predict that the processed complex (mammalian-derived virus) or paucimannose (insect-derived virus) glycan is at position N154 which is not essential for virus production and spread in mosquito or mammalian cells ${ }^{48}$, as the high mannose-type $\mathrm{N}$-glycans have been reported to be present at the N154 residue in DI domain of ZIKV E within the conserved glycosylation motif $\mathrm{N}-\mathrm{X}-\mathrm{S} / \mathrm{T}^{25,49}$. Moreover, Asian strains (including strains circulating in Southeast Asia, and South and Central America) of ZIKV, but not African strains, contain the N154 glycosylation site of the E protein (Supplementary Fig. 1), which has been reported previously ${ }^{50}$. Contrasting the four African and Asian lineage viruses, the Brazilian ZIKV isolate SJRP revealed only partial similarities in N-glycosylation. SJRP was decorated only with non-sialylated N-glycans when generated in both C6/36 and Vero cell lines, however, mostly with sialylated N-glycans in THP1, SNB-19 and JEG-3 cells (Fig. 2-3, Supplementary Fig. 4), suggesting that decoration of ZIKV E with sialylated N-glycans may correlate with diverse tissue tropism of the virus. Taken together, these findings lead us to hypothesize that the "N-glycotype" of the recently emergent Asian strains is mediating the incidence of the new neuropathogenic potential of ZIKV infection.

The results of our study revealed high heterogeneity in structure and composition of the Nglycans linked to ZIKV E produced by both insect and various mammalian cells including monocytes, placental, and neural cell types. The heterogeneity of N-glycan has also been linked to DENV E protein in terms of structure and composition ${ }^{23}$. The N-glycan on DENV E protein that is produced by mammalian cells is a mixture of high-mannose glycan and complex glycan. Mosquito cells, by contrast, provide a mix of high-mannose glycan and paucimannose glycan ${ }^{46}$. It has been shown that ZIKV replication in macaques also restores the absent N-glycosylation site in MR766 strain ${ }^{51}$, suggesting that the passage history (host animal and cell line types) 
262 affects $\mathrm{E}$ protein glycosylation pattern of ZIKV strains. Taken together, our study revealed

263 glycan structures linked to ZIKV E, analyzed by using MS and lectin microarray, are varied in

264 structure and composition depending on ZIKV strains and host cell types.

265 The C6/36, Vero, THP-1, and SNB-19 cells produced ZIKV E N-glycosylation profiles with 266 highly sialylated N-glycans on the E protein of all ZIKV strains, except for MR766 in JEG-3 cell and SJRP in C6/36 and Vero cells where the N-glycosylation profile was mostly restricted to low molecular weight non-sialylated N-glycans. These data suggest that the different contexts of

269 specific host cells could significantly affect the $\mathrm{N}$-glycosylation profile of the ZIKV E of different ZIKV strains. The cell culture media, culture conditions, and sequence of a protein may 271 also significantly influence the quality and relative abundance of N-glycans observed on the cell272 produced glycoproteins. The results of our study also revealed a high heterogeneity in the N273 glycans linked to ZIKV E among different ZIKV strains that are produced by the same host cell.

274 For example, Vero cells produced ZIKV E linked to various N-glycans among ZIKV strains 275 including FRL, IbH, PRVABC59, and MR766. Unlike other flaviviruses, ZIKV E contains a 276 conserved sequence of $\sim$ ten amino acids that surround the N154 glycosylation site. This 277 conserved sequence may affect the glycosylation process, carbohydrate moiety, and attachment 278 site of the ZIKV to host cells ${ }^{18}$. The number and location of glycosylation motifs in the E vary 279 considerably both between and within flaviviruses ${ }^{52}$, altering the pathogenicity of these viruses and the severity of their diseases. The African lineage of ZIKV isolates lack the N-glycosylation 281 site while ZIKV isolates from recent outbreaks in South and Central American regions, that was 282 originated from the epidemy of Asian lineage ZIKV PF-2013 in French Polynesia in 2013, 283 contain the glycosylation site within the viral envelope ${ }^{22}$. Indeed, in a previous study, the 284 recombinant ZIKV strains that lack the glycosylation site were unable to induce mortality in the 
285

286

287

288

289

290

291

292

293

294

295

296

297

298

299

300

301

302

303

304

305

306

307

mouse model ${ }^{22}$. However, recent studies revealed that residues surrounding the ZIKV E protein glycan regulate virus antigenicity, irrespective of the presence of a glycan ${ }^{53}$. The glycosylation of ZIKV E protein does not affect antibody binding to a nearby epitope or its capacity to serve as a neutralization target ${ }^{54}$. Collectively, these findings suggest that ZIKV pathogenicity, but not immunogenicity, might be influenced by the glycosylation of the E protein which occurs posttranslationally in ER of the host cell. This host cell-dependent glycosylation process of ZIKV E could thus have wide ranging implications regarding the pathogenicity and infectivity of the virus. However, future research is required to evaluate the full extent of this circumstance.

In regard to infection of cells, we explored ZIKV interactions with cellular lectins that bind specific types of glycans we identified on the virus. DC-SIGN is a lectin-like molecule, expressed in dendritic cells that can facilitate virus entry through its binding affinity with the glycans on the E protein ${ }^{55}$. N154 glycosylation was found to play a role in ZIKV infection of mammalian cells through this entry factor DC-SIGN ${ }^{48}$. Indeed, the glycosaminoglycan (GAGs) of C-type lectins, including DC-SIGN, have a binding affinity as an attachment factor for host cell entry of ZIKV similar to other pathogenic flaviviruses ${ }^{56}$. We also observed a significant decrease in ZIKV infection in DC-SIGN and L-SIGN expressing cells in the presence of competitive inhibitors rich in mannose. As a result, the presence of monoclonal antibodies to various C-type lectins, the competitive inhibitors rich in mannose, or antiviral gene therapy may be able to reduce ZIKV replication by impairing the binding between viral N-glycan of E protein and GAGs of DC-SIGN. Recent reports suggest that the cells expressing DC-SIGN, such as human dermal fibroblasts, epidermal keratinocytes, and immature dendritic cells, are permissive to the most recent ZIKV isolates. This may have contributed to the epidemic in French Polynesia in 2013 where the cell entry mechanism of ZIKV strain PF-2013 to various cell lines was shown 
308

309

310

311

312

313

314

315

316

317

318

to be mediated by a range of receptors and molecules including DC-SIGN ${ }^{57}$. The accessibility of ZIKV PF-2013 strain, the original strain initiated in the recent outbreaks of ZIKV in South and Central American regions, to different entry cell receptors possibly provides an evolutionary advantage for this recent ZIKV strain to infect a wide range of target cells and evade the immune system of human host. Taken together, these phenomena may partially explain the alterations in the mechanisms of pathogenesis and tissue tropism of these recent ZIKV strains. Further studies are needed to determine the impact of host glycosylation variability on ZIKV pathogenesis and infectivity in vivo.

In conclusion, our findings provide a first-of-its-kind detailed repertoire of $\mathrm{N}$-glycans linked to the ZIKV envelope. To our knowledge, this is the first comprehensive mapping on the glycome of ZIKV E in different physiologically relevant cell lines. Our study showed that the N-glycans linked to ZIKV E produced by both insect and various mammalian cells are highly heterogenous in structure and composition. Both African and Asian lineage strains showed very similar Nglycosylation patterns of ZIKV E. We also noticed that the N-glycan pattern of the ZIKV E depends on not only virus strain characteristics but also on cell line type. We showed that ZIKV infection in DC-SIGN and L-SIGN expressing cells was significantly decreased in the presence of competitive inhibitors rich in mannose, suggesting an important role for these lectin-like molecules as cell entry factors in pathogenesis, emerging new strains of ZIKV and alternative targets for therapeutic development. The results of this study are important in efforts to design therapeutically active antibodies against ZIKV replication and glycomimetic compound-based antivirals. 


\section{Methods}

332

333

334

335

336

337

338

\section{Cell lines and viruses}

The ZIKV stocks were produced in various cell lines derived from human (placenta, brain, and monocytes), monkey, and insects (Supplementary Table 1a), and tittered in Vero (kidney epithelial cells extracted from an African green monkey) cells. ZIKV strains, MR766 (Rhesus/1947/Uganda; BEI Cat. \# NR-50065),PRVABC59 (Human/2015/Puerto Rico; BEI Cat. \# NR-50240), FLR (Human/2015/Colombia; BEI Cat. \# NR-50183), IbH 30656 (Human/1968/Nigeria; BEI Cat. \# NR-50066) were obtained from BEI Zika resources. A ZIKV Brazilian isolate (SJRP-HB-2016-1840 - herein referred to as SJRP) was also obtained from University of Texas Medical Branch (UTMB) Arbovirus reference collection (Supplementary Table 1b). The cells were cultured and maintained in Dulbecco's modified eagle medium (DMEM, Gibco, Life Technologies, Carlsbad CA, USA) supplemented with 10\% heatinactivated fetal bovine serum (FBS, Invitrogen, Carlsbad, CA, USA) at $37^{\circ} \mathrm{C}$ in a $5 \%$ carbon dioxide humidified environment. The mammalian cell lines JEG-3 (human placental choriocarcinoma) and SNB-19 (human brain glioblastoma) were grown on DMEM (1X) supplemented with the 10\% FBS. The LLC-MK2 (kidney epithelial cells extracted from Macaca mulatta monkey) cells were grown on Medium 199 (Biowest, Riverside, MO, USA) supplemented with 1\% horse serum (Invitrogen, CA, USA). The THP-1 (human monocytic cells) cells were grown on Roswell Park Memorial Institute (RPMI) medium (Gibco, CA, USA) supplemented with 10\% FBS and $0.05 \mathrm{mM} \beta$-mercaptoethanol (Sigma-Aldrich, St. Louis, MO, USA). The mosquito C6/36 (Aedes albopictus clone) cells were grown on minimum essential medium (MEM, Gibco, Life Technologies, CA, USA) supplemented with 10\% FBS. All cell culture media were supplemented with 1\% penicillin-streptomycin (Gibco, CA, USA) and 
354 maintained at $37{ }^{\circ} \mathrm{C}$ in a $5 \%$ carbon dioxide humidified environment, except the C6/36 cells

355 which were maintained at $28^{\circ} \mathrm{C}$.

\section{Virus infection}

357 The various ZIKV strains, such as African strain (MR766 and IbH), Asian strains (PRVABC59 358 and FLR), and a primary isolate from Brazil in 2016 (SJRP) at multiplicity of infection (MOI) of 3591 was adsorbed onto cells. After $90 \mathrm{~min}$, in serum-free medium with rocking every $15 \mathrm{~min}$, the 360 inoculum was removed; the C6/36, Vero, LLC-MK2, SNB-19, THP-1, and JEG-3 cells were 361 maintained in respective medium containing 2\% FBS containing respective medium. All the 362 cells lines were maintained at $37^{\circ} \mathrm{C}$ in a $\mathrm{CO} 2$ incubator (except the $\mathrm{C6} / 36$ cells which were maintained at $28^{\circ} \mathrm{C}$ ). For the large-scale virus production, all cells were maintained at $37^{\circ} \mathrm{C}$ in

$364 \mathrm{CO} 2$ incubator for 3-4 days (except C6/36 cells which were maintained at $28{ }^{\circ} \mathrm{C}$ for $5-7$ days) 365 until cytopathic effects were observed.

\section{Immunofluorescence assay}

367 The C6/36, Vero, and SNB-19 cells were grown on cover slips and mock-infected or infected 368 with various strains of ZIKV. After 24 hours post infection, cells were processed for indirect 369 immunofluorescence assay using the double-labelling method. Briefly, cells were fixed in $4 \%$ 370 paraformaldehyde and processed for immunofluorescence assay. The cells were blocked and 371 permeabilized using 5\% goat serum (Sigma-Aldrich, St. Louis, MO, USA) with 0.5\% Triton X-

372100 (Sigma-Aldrich, St. Louis, MO, USA). The cells were then immunoassayed using rabbit 373 polyclonal antibody (1:1000) against the E protein of ZIKV (Genetex Inc., Irvine, CA, USA), in 374 a combination with ER, using ER ID dye as per manufacturer's instructions (Enzo Life Sciences, 375 Inc, Farmingdale, NY, USA) and the cell nuclei (1:2000), using Hoechst stain (Invitrogen, 
Rockford, IL, USA) for 15 min. Subsequently, Alexa Fluor 594-conjugated secondary antibody

377 (1:2000) (Invitrogen, Rockford, IL, USA) was added onto cells; and images were captured under 40X using Operetta High-Content Imaging System (PerkinElmer, Waltham, MA, USA).

\section{Purification of ZIKV virus and isolation of $E$ protein}

A large stock of ZIKV viruses were purified using sucrose cushion in ultracentrifugation as described in previous methods ${ }^{26}$. Briefly, culture supernatants were centrifuged at $6,000 \mathrm{rpm}$ for $10 \mathrm{~min}$, and a $0.45 \mu \mathrm{m}$ filter was used to remove cell debris. Virus-containing filtrate was layered onto $20 \%(\mathrm{w} / \mathrm{v})$ sucrose and subjected to ultracentrifugation at $100,715 \times \mathrm{g}, 4{ }^{\circ} \mathrm{C}$ for 3.5 hours. $1 \mathrm{mM}$ EDTA (ethylene diamine tetra acetic acid)). The purified virions were then resolved on gradient $4-12 \%$ bis-tris protein gels. The presence of ZIKV glycoprotein E was confirmed by western blot analysis, and confirmed band was excised for MS analysis.

\section{Lectin microarray}

For the lectin array analysis, the cells (C6/36 cells, Vero cells, SNB-19 cells, and JEG-3 cells) were grown to 60-70 \% confluency. Various ZIKV strains, such as African strain (MR766 and $\mathrm{IbH}$ ), Asian strains (PRVABC59 and FLR) and the primary Brazilian isolate SJRP, at MOI of 1 were then added onto cells for $90 \mathrm{~min}$, in serum-free medium. After $90 \mathrm{~min}$, the inoculum was removed; and cells continued to culture in their respective culture conditions. The cells were

394 harvested using cell scrapper, made into a single cell suspension, and counted using cell countess

395 (Invitrogen, Carlsbad, CA, USA). The lectin microarray enabled sensitive analysis of multiple 396 glycan structures (45 structures) by employing a panel of immobilized lectins with known glycan

397 binding specificity ${ }^{27-35}$. Cell-surface proteins were purified using Mem-PER TM Plus Membrane 
Protein Extraction Kit (Thermo Fisher Scientific, Rockford, IL, USA). Isolated proteins were labeled with $\mathrm{Cy} 3$ dye and hybridized to a lectin microarray ${ }^{27-35}$. The resulting lectin chips were scanned for fluorescence intensity on each lectin-coated spot using an evanescent-field fluorescence scanner. Data were normalized using the global normalization method.

\section{N-glycan preparation}

403 Gel bands stained with Coomassie Blue were excised and transferred into clean tubes. Then, 200 $404 \mu \mathrm{l}$ of $50 \mathrm{mM}$ ammonium bicarbonate (AMBIC) (Sigma-Aldrich, St Louis, MO, USA) and $200 \mu 1$ 405 of acetonitrile (Sigma-Aldrich, St. Louis, MO, USA) were added to the samples, mixed 406 thoroughly and incubated at room temperature (RT) for $5 \mathrm{~min}$. The supernatants were then 407 discarded, and the washing step was repeated once more. The gel pieces were dried with a 408 vacuum centrifuge for $10 \mathrm{~min}$. Then $200 \mu \mathrm{l}$ of a $10 \mathrm{mM}$ DTT (1,4-Dithiothreitol, Sigma-Aldrich, 409 St. Louis, MO, USA) solution was added and incubated at $50{ }^{\circ} \mathrm{C}$ for $30 \mathrm{~min}$. The DTT solution 410 was discarded, and the samples were briefly washed with $200 \mu$ l of acetonitrile solution. Again, 411 the samples were dried with a vacuum centrifuge for $10 \mathrm{~min}$.

412 After drying, the samples were incubated with $200 \mu \mathrm{l}$ of a $55 \mathrm{mM}$ IAA (Iodoacetamide, Sigma413 Aldrich, St. Louis, MO, USA) solution for $30 \mathrm{~min}$ in the dark at RT. The IAA solution was then 414 discarded, and the samples were washed with $500 \mu \mathrm{l}$ of $50 \mathrm{mM}$ AMBIC for $15 \mathrm{~min}$ at RT, 415 followed by $5 \mathrm{~min}$ incubation with $200 \mu \mathrm{l}$ of acetonitrile. The samples were dried once again 416 with a vacuum centrifuge for $10 \mathrm{~min}$, before adding $500 \mu \mathrm{l}$ of $50 \mathrm{mM}$ AMBIC containing $10 \mu \mathrm{g}$ 417 of TPCK-treated trypsin (Sigma-Aldrich, St. Louis, MO, USA). After incubating overnight at 37 $418{ }^{\circ} \mathrm{C}$, the trypsin digestion was terminated by boiling the sample for $3 \mathrm{~min}$. 
419 The supernatants were recovered and collected in a clean glass tube, before carrying out two 420 sequential washes with $200 \mu \mathrm{l}$ of $50 \mathrm{mM}$ AMBIC, vortexed for $15 \mathrm{~min} ; 200 \mu 1$ of $50 \%$ 421 acetonitrile in $50 \mathrm{mM}$ AMBIC, vortexed for $15 \mathrm{~min}$; and $200 \mu \mathrm{l}$ of acetonitrile, vortexed for 15 422 min. For each sample, all washes were collected, pooled in the same glass tube that was 423 previously used, and then lyophilized. The dried materials were resuspended in $200 \mu 1$ of $50 \mathrm{mM}$ 424 AMBIC. $1 \mu 1$ of PNGaseF (New England Biolabs, Ipswich, MA, USA) was added to this for an 425 overnight incubation at $37^{\circ} \mathrm{C}$. Two drops of 5\% acetic acid (Fisherbrand, Waltham, MA, USA) 426 were added to stop the enzymatic reaction before purifying the released N-glycan over a C18 427 Sep-Pak (50 mg) column (Waters, Milford, MA, USA) that was conditioned with 1 column 428 volume (CV) of methanol (Sigma-Aldrich, St. Louis, MO, USA), 1 CV of 5\% acetic acid, 1 CV 429 of 1-propanol (Sigma-Aldrich), and $1 \mathrm{CV}$ of 5\% acetic acid. The C18 column was washed with 3 $\mathrm{ml}$ of 5\% acetic acid, flow through; and wash fractions were collected, pooled, and lyophilized.

\section{Permethylation of N-glycan}

432 Lyophilized N-glycan samples were incubated with $1 \mathrm{ml}$ of a DMSO (Dimethyl Sulfoxide; 433 Sigma)-NaOH (Sigma-Aldrich, St. Louis, MO, USA) slurry solution and $500 \mu$ l of methyl iodide 434 (Sigma-Aldrich, St. Louis, MO, USA) for 20-30 min under vigorous shaking at RT. $1 \mathrm{ml}$ of 435 Milli-Q water was added to stop the reaction. $1 \mathrm{ml}$ of chloroform (Sigma-Aldrich, St. Louis, MO, USA) was added to purify the permethylated N-glycan. $3 \mathrm{ml}$ of Milli-Q water were added to 437 wash the chloroform fractions, and the mixture was briefly vortexed. The water was discarded by 438 additional centrifugation. This wash step was repeated 3 times. The chloroform fraction was 439 dried before being redissolved in $200 \mathrm{ml}$ of $50 \%$ methanol. This was then loaded into a 440 conditioned (1 CV methanol, 1 CV Milli-Q water, 1 CV acetonitrile (Sigma-Aldrich, St. Louis, 441 MO, USA), and 1 CV Milli-Q Water) C18 Sep-Pak (200 mg) column. The C18 column was 
442 washed with $6 \mathrm{ml}$ of $15 \%$ acetonitrile and then eluted with $6 \mathrm{ml}$ of $50 \%$ acetonitrile. The eluted

443 fraction was lyophilized and then redissolved in $10 \mu 1$ of $75 \%$ methanol from which $1 \mu 1$ was

444 mixed with $1 \mu 1$ DHB (2,5-dihydroxybenzoic acid (Sigma-Aldrich, St. Louis, MO, USA))

$445(5 \mathrm{mg} / \mathrm{ml}$ in $50 \%$ acetonitrile with $0.1 \%$ trifluoroacetic acid (Sigma-Aldrich, St. Louis, MO, 446 USA)) and spotted on a MALDI polished steel target plate (Bruker Daltonics, Bremen, 447 Germany).

\section{MS data acquisition and analyses}

449 MS data were acquired on a Bruker UltraFlex II MALDI-TOF Mass Spectrometer instrument.

450 The reflective positive mode was used, and data were recorded between $500 \mathrm{~m} / \mathrm{z}$ and $6000 \mathrm{~m} / \mathrm{z}$.

451 For each MS N-glycan profile, the aggregation of 20,000 laser shots or more were considered for

452 data extraction. Mass signals of a signal/noise ratio of at least 4 were considered, and only MS

453 signals matching an N-glycan composition were considered for further analysis. Subsequent MS

454 post-data acquisition analysis was made using $\mathrm{mMass}^{36}$. The relative abundance of each $\mathrm{N}-$

455 glycans identified on ZIKV $\mathrm{E}$ in each experimental condition was calculated based on the 456 absolute intensity of the first isotopic peak of a given $\mathrm{N}$-glycan relative to the sum of all N457 glycan intensities.

458 Assays to evaluate the functional importance of $\mathbf{N}$-linked glycans

459 In inhibition experiments, 3T3, 3T3-DCSIGN and 3T3-L-SIGN cells were incubated with 200

$460 \mu \mathrm{g} / \mathrm{mL}$ of yeast mannan (Sigma- Aldrich, St. Louis, MO, USA) or $0.5 \mathrm{mM}$ EDTA at $37{ }^{\circ} \mathrm{C}$ for

$46130 \mathrm{~min}$. Various strains of Zika viruses $(\mathrm{MOI}=1)$ were preincubated with same concentration of

462 inhibitor and adsorbed on pretreated cells. The mannan treatment was maintained constant

463 throughout duration of infection. Whereas, the EDTA and virus containing medium was removed 
464 after $2 \mathrm{~h}$ and continued the infection with the fresh medium. The infected cells were detected

465 using viral E protein antibody, and the percent of infected cells was assessed by an

466 immunofluorescence assay using Operetta High-Content Imaging System (PerkinElmer, Inc.,

467 Waltham, MA, USA).

468 Statistical Analysis

469 Spearman's Rank Order Correlations were conducted using GraphPad Prism release 7.0

470 (GraphPad Software, San Diego, CA, USA).

471 


\section{$472 \quad$ References}

4731 Brooks, J. T. et al. Update: Interim Guidance for Prevention of Sexual Transmission of 474 Zika Virus - United States, July 2016. MMWR Morb Mortal Wkly Rep 65, 745-747, 475 doi:10.15585/mmwr.mm6529e2 (2016).

4762 Burd, I. \& Griffin, D. The chasm between public health and reproductive research: what 477 history tells us about Zika virus. J Assist Reprod Genet 33, 439-440, doi:10.1007/s10815-

478 016-0687-3 (2016).

4793 Slavov, S. N., Otaguiri, K. K., Kashima, S. \& Covas, D. T. Overview of Zika virus 480 (ZIKV) infection in regards to the Brazilian epidemic. Braz J Med Biol Res 49, e5420, doi:10.1590/1414-431X20165420 (2016).

4824 Wikan, N. \& Smith, D. R. Zika virus: history of a newly emerging arbovirus. Lancet Infect Dis 16, e119-126, doi:10.1016/S1473-3099(16)30010-X (2016).

4845 Aubry, M. et al. Zika Virus Seroprevalence, French Polynesia, 2014-2015. Emerg Infect 485 Dis 23, 669-672, doi:10.3201/eid2304.161549 (2017).

4866 Oehler, E. et al. Zika virus infection complicated by Guillain-Barre syndrome--case 487 report, French Polynesia, December 2013. Euro Surveill 19 (2014).

4887 Lednicky, J. et al. Zika Virus Outbreak in Haiti in 2014: Molecular and Clinical Data. 489 PLoS Negl Trop Dis 10, e0004687, doi:10.1371/journal.pntd.0004687 (2016).

4908 Avelino-Silva, V. I. \& Martin, J. N. Association between Guillain-Barre syndrome and 491 Zika virus infection. Lancet 387, 2599, doi:S0140-6736(16)30843-1 [pii] 
4939 Brito, C. A. \& Cordeiro, M. T. One year after the Zika virus outbreak in Brazil: from 494 hypotheses to evidence. Rev Soc Bras Med Trop 49, 537-543, doi:10.1590/0037-86820328-2016 (2016).

49610 Alvarado, M. G. \& Schwartz, D. A. Zika Virus Infection in Pregnancy, Microcephaly, 497 and Maternal and Fetal Health: What We Think, What We Know, and What We Think We Know. Arch Pathol Lab Med 141, 26-32, doi:10.5858/arpa.2016-0382-RA (2017).

49911 Blazquez, A. B. \& Saiz, J. C. Neurological manifestations of Zika virus infection. World 500 J Virol 5, 135-143, doi:10.5501/wjv.v5.i4.135 (2016).

50112 Mlakar, J. et al. Zika Virus Associated with Microcephaly. N Engl J Med 374, 951-958, 502 doi:10.1056/NEJMoa1600651 (2016).

50313 Schuler-Faccini, L. et al. Possible Association Between Zika Virus Infection and 504 Microcephaly - Brazil, 2015. MMWR Morb Mortal Wkly Rep 65, 59-62, 505 doi:10.15585/mmwr.mm6503e2 (2016).

50614 Sarno, M. et al. Zika Virus Infection and Stillbirths: A Case of Hydrops Fetalis, 507 Hydranencephaly and Fetal Demise. PLoS Negl Trop Dis 10, e0004517, 508 doi:10.1371/journal.pntd.0004517 (2016).

50915 Saiz, J. C. et al. Zika Virus: the Latest Newcomer. Front Microbiol 7, 496, 510 doi:10.3389/fmicb.2016.00496 (2016).

$511 \quad 16$ Zhang, X. et al. Structures and Functions of the Envelope Glycoprotein in Flavivirus $512 \quad$ Infections. Viruses 9, doi:10.3390/v9110338 (2017).

51317 Kostyuchenko, V. A. et al. Structure of the thermally stable Zika virus. Nature 533, 425428, doi:nature 17994 [pii] 
51618 Sirohi, D. et al. The 3.8 A resolution cryo-EM structure of Zika virus. Science 352, 467470, doi:science.aaf5316 [pii]

$518 \quad 10.1126 /$ science.aaf5316 (2016).

51919 Barba-Spaeth, G. et al. Structural basis of potent Zika-dengue virus antibody cross$520 \quad$ neutralization. Nature 536, 48-53, doi:10.1038/nature18938 (2016).

52120 Routhu, N. K. \& Byrareddy, S. N. Host-Virus Interaction of ZIKA Virus in Modulating

522 Disease Pathogenesis. J Neuroimmune Pharmacol 12, 219-232, doi:10.1007/s11481-017$523 \quad 9736-7(2017)$.

52421 Aebi, M. N-linked protein glycosylation in the ER. Biochim Biophys Acta 1833, 2430$525 \quad$ 2437, doi:10.1016/j.bbamcr.2013.04.001 (2013).

52622 Annamalai, A. S. et al. Zika Virus Encoding Non-Glycosylated Envelope Protein is Attenuated and Defective in Neuroinvasion. J Virol, doi:10.1128/JVI.01348-17 (2017).

52823 Lei, Y. et al. Characterization of N-Glycan Structures on the Surface of Mature Dengue 2 Virus Derived from Insect Cells. PLoS One 10, e0132122, doi:10.1371/journal.pone.0132122 (2015).

53124 Mossenta, M., Marchese, S., Poggianella, M., Slon Campos, J. L. \& Burrone, O. R. Role of N-glycosylation on Zika virus E protein secretion, viral assembly and infectivity.

53425 Fontes-Garfias, C. R. et al. Functional Analysis of Glycosylation of Zika Virus Envelope 535 Protein. Cell Rep 21, 1180-1190, doi:10.1016/j.celrep.2017.10.016 (2017).

53626 Coelho, S. V. A. et al. Development of standard methods for Zika virus propagation, 537 titration, and purification. $J$ Virol Methods 246, 65-74, doi:10.1016/j.jviromet.2017.04.011 (2017). 
53927 Chen, P. et al. Identification of N-glycan of alpha-fetoprotein by lectin affinity microarray. J Cancer Res Clin Oncol 134, 851-860, doi:10.1007/s00432-008-0357-7

541 (2008).

54228 Chen, S., Zheng, T., Shortreed, M. R., Alexander, C. \& Smith, L. M. Analysis of cell surface carbohydrate expression patterns in normal and tumorigenic human breast cell lines using lectin arrays. Anal Chem 79, 5698-5702, doi:10.1021/ac070423k (2007).

545

546

547

548

549

550

551

552

553

554

555

556

557

558

559

560

561

29 Matsuda, A. et al. Development of an all-in-one technology for glycan profiling targeting formalin-embedded tissue sections. Biochem Biophys Res Commun 370, 259-263, doi:10.1016/j.bbrc.2008.03.090 (2008).

30 Angeloni, S. et al. Glycoprofiling with micro-arrays of glycoconjugates and lectins. Glycobiology 15, 31-41, doi:10.1093/glycob/cwh143 (2005).

31 Carlsson, J., Mecklenburg, M., Lundstrom, I., Danielsson, B. \& Winquist, F. Investigation of sera from various species by using lectin affinity arrays and scanning ellipsometry. Analytica chimica acta 530, 167-171, doi:10.1016/j.aca.2004.09.022 (2005).

32 Fromell, K., Andersson, M., Elihn, K. \& Caldwell, K. D. Nanoparticle decorated surfaces with potential use in glycosylation analysis. Colloids Surf B Biointerfaces 46, 84-91, doi:10.1016/j.colsurfb.2005.06.017 (2005).

33 Kuno, A. et al. Evanescent-field fluorescence-assisted lectin microarray: a new strategy for glycan profiling. Nat Methods 2, 851-856, doi:10.1038/nmeth803 (2005).

34 Pilobello, K. T., Krishnamoorthy, L., Slawek, D. \& Mahal, L. K. Development of a lectin microarray for the rapid analysis of protein glycopatterns. Chembiochem 6, 985-989, doi:10.1002/cbic.200400403 (2005). 
56235 Zheng, T., Peelen, D. \& Smith, L. M. Lectin arrays for profiling cell surface carbohydrate 563 expression. J Am Chem Soc 127, 9982-9983, doi:10.1021/ja0505550 (2005).

56436 Strohalm, M., Kavan, D., Novak, P., Volny, M. \& Havlicek, V. mMass 3: a cross565 platform software environment for precise analysis of mass spectrometric data. Anal 566 Chem 82, 4648-4651, doi:10.1021/ac100818g (2010).

56737 van Liempt, E. et al. Specificity of DC-SIGN for mannose- and fucose-containing 568 glycans. FEBS Lett 580, 6123-6131, doi:10.1016/j.febslet.2006.10.009 (2006).

56938 Feinberg, H., Mitchell, D. A., Drickamer, K. \& Weis, W. I. Structural basis for selective 570 recognition of oligosaccharides by DC-SIGN and DC-SIGNR. Science 294, 2163-2166, doi:10.1126/science.1066371 (2001).

57239 Alvarez, C. P. et al. C-type lectins DC-SIGN and L-SIGN mediate cellular entry by 573 Ebola virus in cis and in trans. J Virol 76, 6841-6844 (2002).

57440 Baribaud, F., Pohlmann, S., Leslie, G., Mortari, F. \& Doms, R. W. Quantitative 575 expression and virus transmission analysis of DC-SIGN on monocyte-derived dendritic cells. J Virol 76, 9135-9142 (2002).

57741 Lozach, P. Y. et al. Dendritic cell-specific intercellular adhesion molecule 3-grabbing non-integrin (DC-SIGN)-mediated enhancement of dengue virus infection is independent of DC-SIGN internalization signals. $J$ Biol Chem 280, 23698-23708,

58142 Zhang, P. et al. Challenges of glycosylation analysis and control: an integrated approach 582 to producing optimal and consistent therapeutic drugs. Drug Discov Today 21, 740-765, doi:10.1016/j.drudis.2016.01.006 (2016). 
58443 Cruz, E., Cain, J., Crossett, B. \& Kayser, V. Site-specific glycosylation profile of 585 influenza A (H1N1) hemagglutinin through tandem mass spectrometry. Hum Vaccin Immunother 14, 508-517, doi:10.1080/21645515.2017.1377871 (2018).

58744 Vigerust, D. J. \& Shepherd, V. L. Virus glycosylation: role in virulence and immune 588 interactions. Trends Microbiol 15, 211-218, doi:10.1016/j.tim.2007.03.003 (2007).

58945 Lee, E., Leang, S. K., Davidson, A. \& Lobigs, M. Both E protein glycans adversely affect 590 dengue virus infectivity but are beneficial for virion release. J Virol 84, 5171-5180, doi:10.1128/JVI.01900-09 (2010).

59246 Hacker, K., White, L. \& de Silva, A. M. N-linked glycans on dengue viruses grown in mammalian and insect cells. J Gen Virol 90, 2097-2106, doi:10.1099/vir.0.012120-0 (2009).

595

47 Go, E. P. et al. Comparative Analysis of the Glycosylation Profiles of MembraneAnchored HIV-1 Envelope Glycoprotein Trimers and Soluble gp140. J Virol 89, 82458257, doi:10.1128/JVI.00628-15 (2015).

$598 \quad 48$ Danyang Gong et al. High-Throughput Fitness Profiling of Zika Virus E Protein Reveals 599 Different Roles for Glycosylation during Infection of Mammalian and Mosquito Cells. iScience 1, 97-111 (2018).

60149 Yap, S. S. L., Nguyen-Khuong, T., Rudd, P. M. \& Alonso, S. Dengue Virus 602 Glycosylation: What Do We Know? Front Microbiol 8, 1415, 603 doi:10.3389/fmicb.2017.01415 (2017).

60450 May, M. \& Relich, R. F. A Comprehensive Systems Biology Approach to Studying Zika 605 Virus. PLoS One 11, e0161355, doi:10.1371/journal.pone.0161355 (2016). 
60651 Aliota, M. T. et al. Heterologous Protection against Asian Zika Virus Challenge in 607 Rhesus Macaques. PLoS Negl Trop Dis 10, e0005168, doi:10.1371/journal.pntd.0005168

608 (2016).

60952 Ishak, H., Takegami, T., Kamimura, K. \& Funada, H. Comparative sequences of two type 610 1 dengue virus strains possessing different growth characteristics in vitro. Microbiol Immunol 45, 327-331 (2001).

61253 Goo, L. et al. The Zika virus envelope protein glycan loop regulates virion antigenicity. Virology 515, 191-202, doi:10.1016/j.virol.2017.12.032 (2018).

61454 Chambers, M. T., Schwarz, M. C., Sourisseau, M., Gray, E. S. \& Evans, M. J. Probing 615 Zika Virus Neutralization Determinants with Glycoprotein Mutants Bearing Linear 616 Epitope Insertions. J Virol 92, doi:10.1128/JVI.00505-18 (2018).

61755 Cambi, A. et al. Microdomains of the C-type lectin DC-SIGN are portals for virus entry 618 into dendritic cells. J Cell Biol 164, 145-155, doi:10.1083/jcb.200306112 (2004).

61956 Kim, S. Y. et al. Interaction of Zika Virus Envelope Protein with Glycosaminoglycans. $620 \quad$ Biochemistry 56, 1151-1162, doi:10.1021/acs.biochem.6b01056 (2017).

62157 Hamel, R. et al. Biology of Zika Virus Infection in Human Skin Cells. J Virol 89, 8880-

622 8896, doi:JVI.00354-15 [pii]

623

10.1128/JVI.00354-15 (2015).

624

625

626

627 

644 USA

645

\section{Acknowledgments}

We thank Russell Jaffe and Robin Taylor for editorial assistance. We acknowledge Krishna Kota (USAMRIID) for his help with Operetta high-content imaging system. We thank Dr. Nikos Vasilakis (UTMB) for kindly providing Zika Brazilian isolate SJRP-HB-2016-1840. This work is supported in part by R01AI113883, Nebraska Neuroscience Alliance Endowed Fund Award to SNB, and the National Center for Functional Glycomics Grant P41GM103694 to RDC.

\section{Author information}

\section{Affiliations}

Department of Pharmacology and Experimental Neuroscience, University of Nebraska Medical Center, Omaha, NE 68198-5800, USA

Nanda Kishore Routhu, Mehdi R. M. Bidokhti \& Siddappa N. Byrareddy

Department of Genetics, Cell Biology and Anatomy, University of Nebraska Medical Center, Omaha, NE 68198-5805, USA; Department of Biochemistry and Molecular Biology, University of Nebraska Medical Center, Omaha, NE 68198-5805, USA

Siddappa N. Byrareddy

Beth Israel Deaconess Medical Center, National Center for Functional Glycomics, Boston, MA,

Sylvain D. Lehoux \& Richard D. Cummings

Beth Israel Deaconess Medical Center Glycomics Core, Boston, MA, USA; 6The Wistar Institute, Philadelphia, PA, USA 
648 Sylvain D. Lehoux \& Emily A. Rouse

649 Department of Pathology and Microbiology, University of Nebraska Medical Center, Omaha, 650 NE, USA.

651 St Patrick Reid

652 The Wistar Institute, Philadelphia, PA, USA

653 Leila B. Giron, Alit Anzurez \& Mohamed Abdel-Mohsen

654

655

\section{Author Contributions}

656 N.K.R. designed, performed, analyzed the data, and drafted the manuscript. S.N.B. designed and 657 supervised the study, contributed to data analysis, and edited the manuscript. S.D.L., and E.A.R.

658 performed the MS analyses. S.D.L., E.A.R., and R.D.C. analyzed the MS data. M.A.M., and 659 L.B.G., performed the lectin array. M.R.M.B., and P.C.R. helped in manuscript editing and 660 interpretation. All authors provided critical feedback and helped shape the research, analysis and 661 manuscript editing.

662 Competing interests

663 The authors declare no competing interests.

664 Corresponding author

665 Correspondence to Siddappa N. Byrareddy.

\section{Supplementary Information (Word file)}




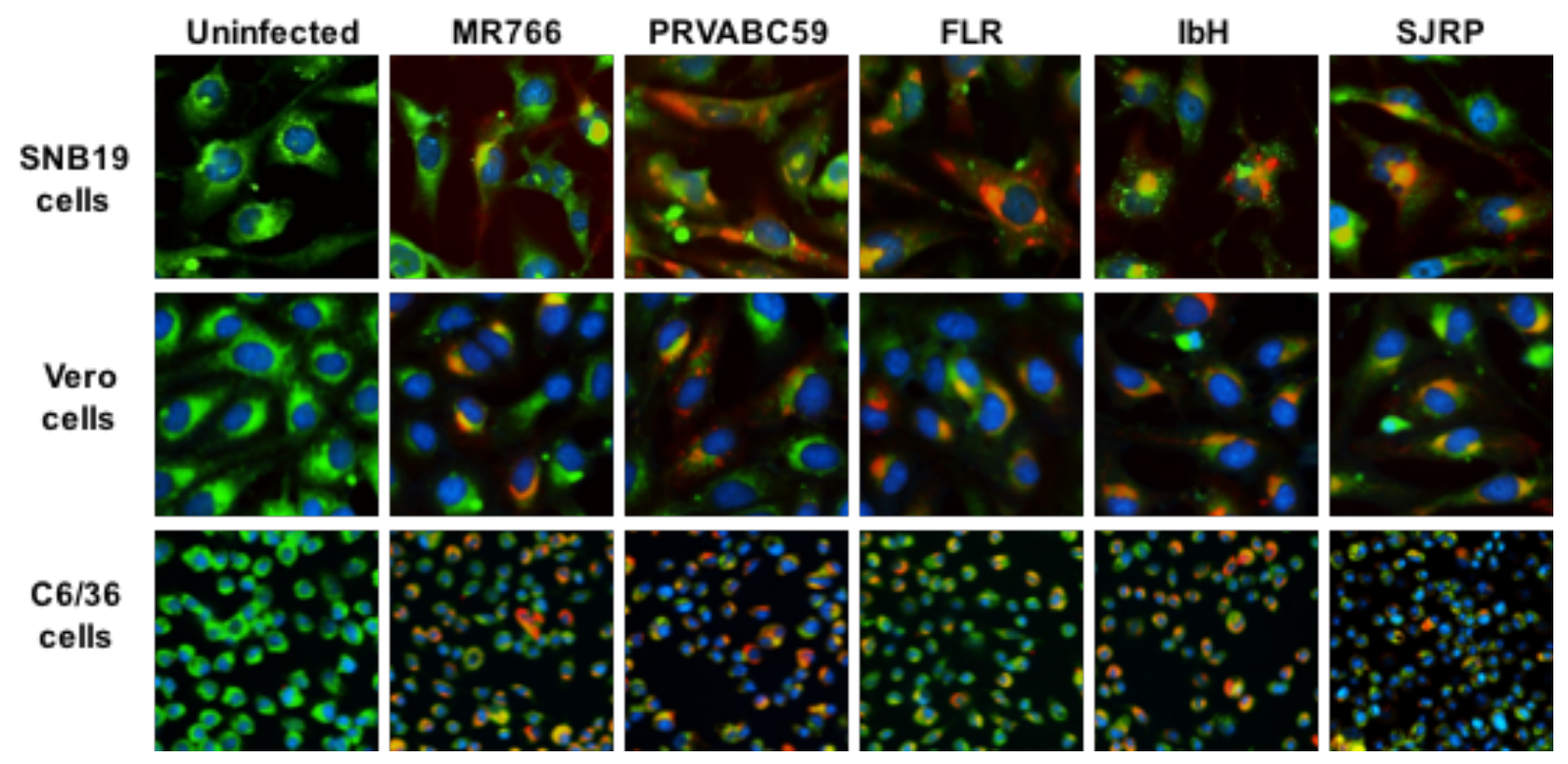

672 Fig. 1. Analyzing the ER-localization of the ZIKV E glycoprotein in infected cells. 673 Immunofluorescence images of SNB-19 cells, Vero cells, and C6/36 cells that were infected with 674 0.1 MOI of ZIKV (MR766, PRVABC59, FLR, IbH, and SJRP). After 24 hours, the cells were 675 fixed, permeabilized, and stained for E protein using a monoclonal anti-flavivirus group antigen 676 antibody (clone D1-4G2-4-15) (red), ER using ER ID dye (green), and cell nuclei using Hoechst 677 stain (blue). No red fluorescence is observed in control cells.

678 
African-lineage

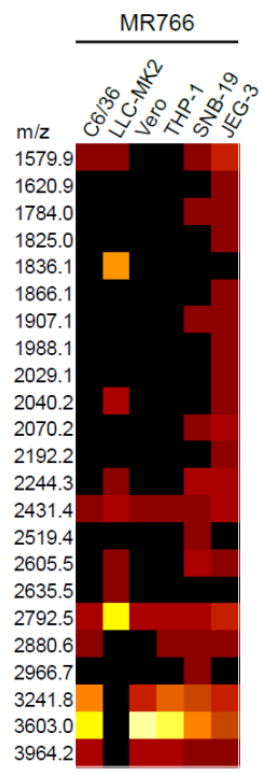

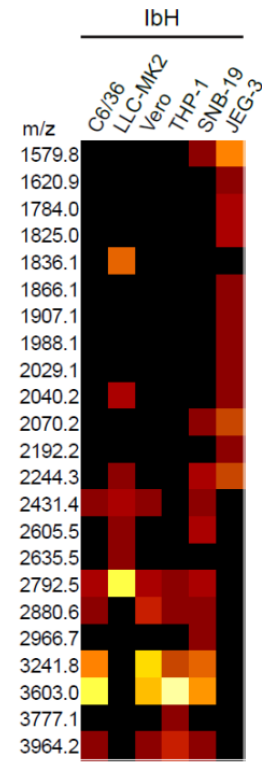

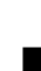

Asian-lineage

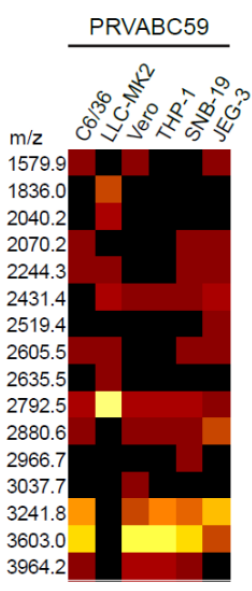

Relative Abundance
Brazilian isolate

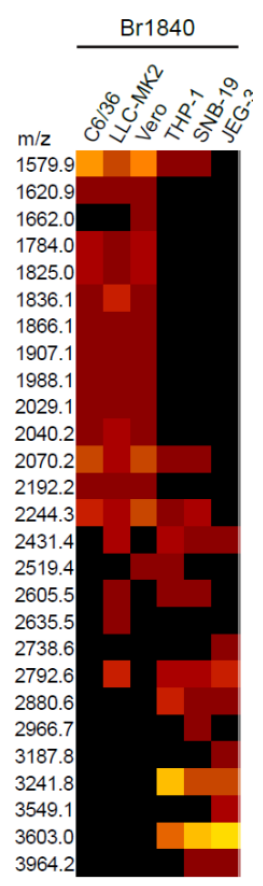

Fig. 2. Heatmap presentation of N-glycans of glycoprotein E of purified ZIKV. ZIKVs (MR766, $\mathrm{IbH}$, PRVABC59, FLR, and SJRP) produced from various cell types (C6/36, LLC-MK2, Vero, THP-1, SNB-19, and JEG-3 cells) were purified using sucrose cushion ultracentrifugation and resolved on SDS-PAGE. The presence of glycoprotein E was confirmed through western blotting. Glycoprotein E was then excised and subjected to N-glycan release, followed by MS identification. The N-glycans identified through this method are represented in heatmap format. 


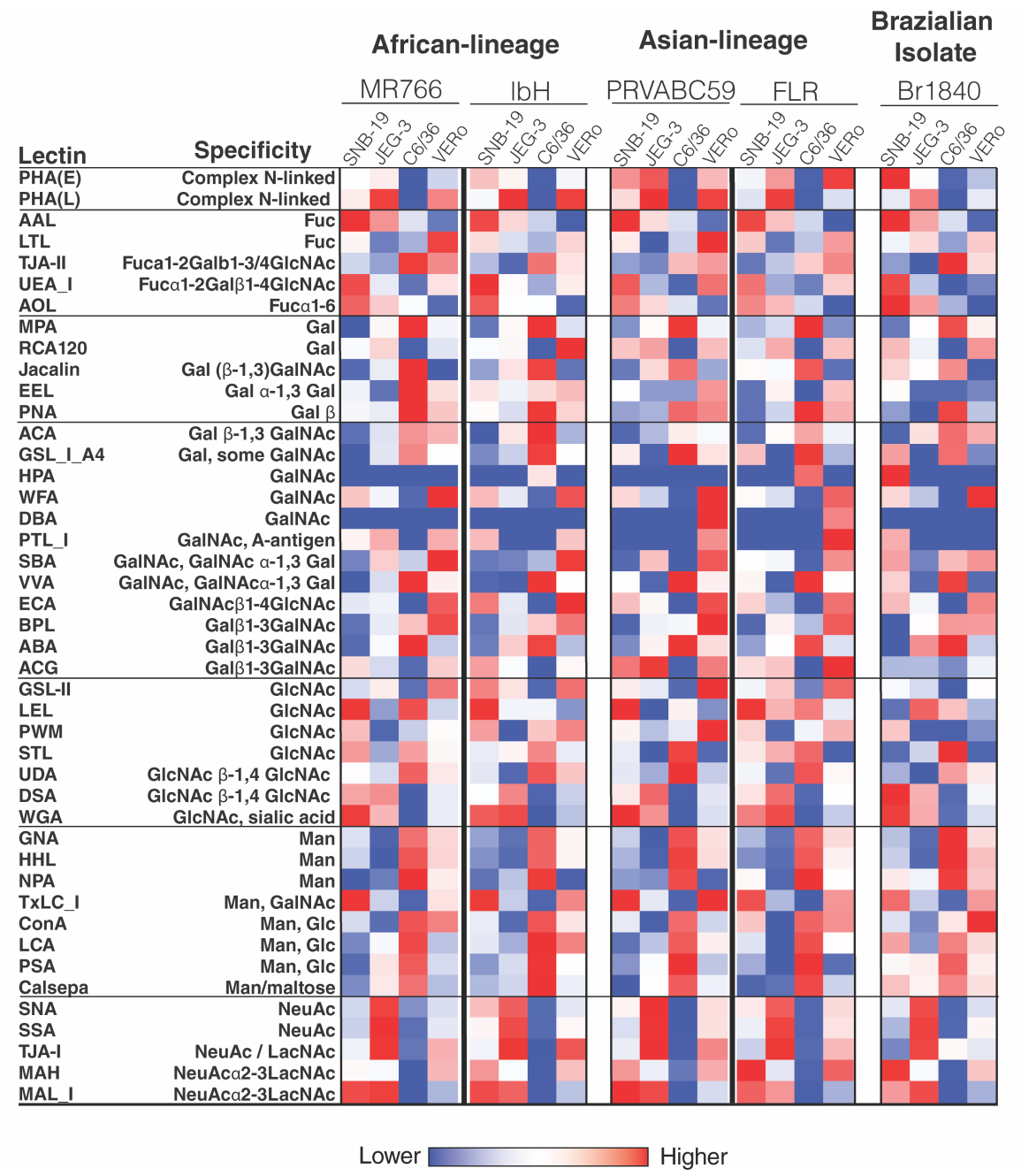

697 Fig. 3. Glycomic profiling of ZIKV-infected and uninfected cell membrane using a lectin microarray. A lectin microarray (45 structures) was used to analyze the glycans associated to cell-surface proteins in both ZIKV-infected and uninfected cells. The different cell types (SNB19, JEG-3, C6/36 and Vero cells) were either mock-infected or infected with distantly related ZIKV strains (MR766, IbH, PRVABC59, FLR, and SJRP). The cell-surface proteins were later extracted using the membrane protein extraction kit, labeled with $\mathrm{Cy} 3$ dye and hybridized to a lectin microarray. The resulting lectin chip was scanned for fluorescence intensity on each lectincoated spot using an evanescent-field fluorescence scanner. The data were normalized using the global normalization method. The heatmap format was used to present the glycan profiles identified using this method. Man represents high-mannose, GalNAc represents Nacetylgalactosamine, GlcNAc represents N-acetylglucosamine, NeuAc represents N- 

fucose.

A

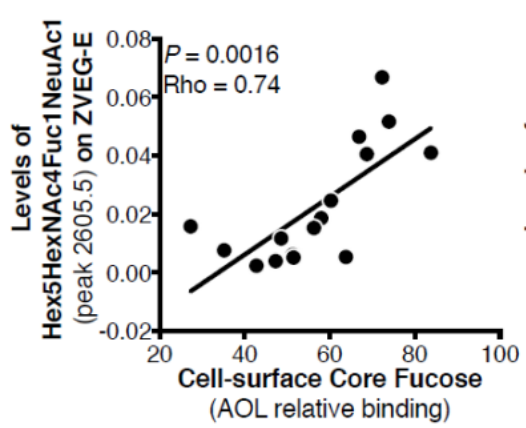

B

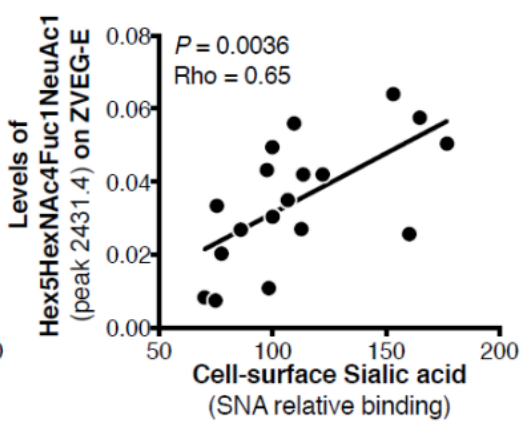

C

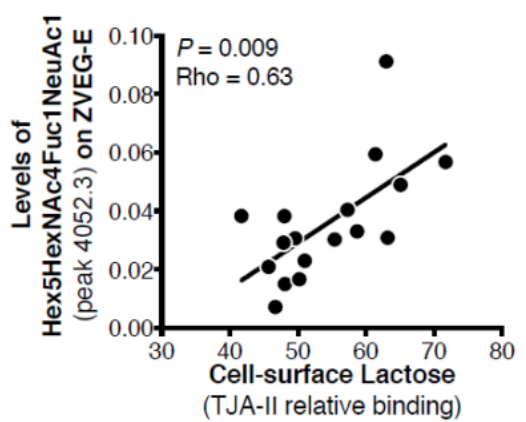

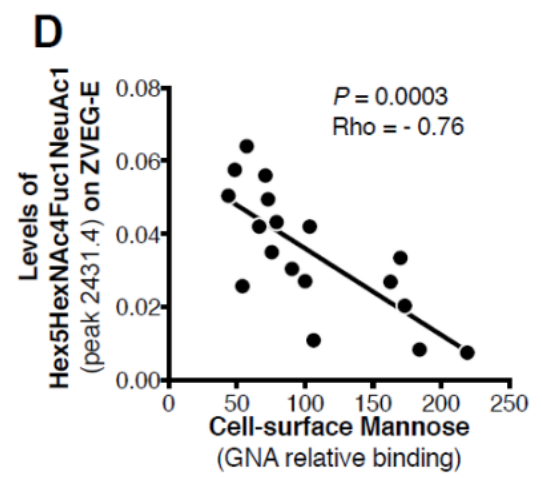

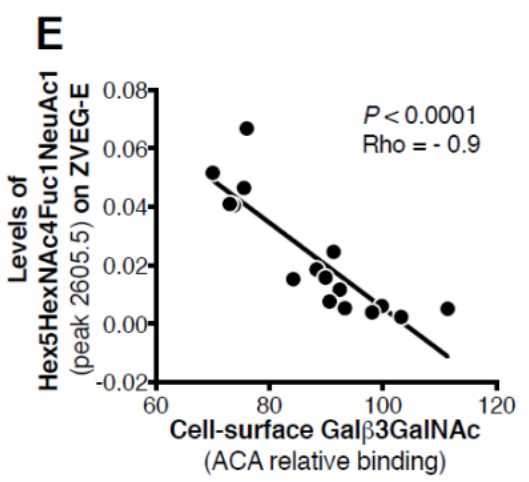

712 Fig. 4. Comparison of glycan profiles identified by MALDI-TOF-MS and by lectin microarray.

713 A significant linear correlation was confirmed between the cell-surface glycans identified by the 714 lectin microarray (fucose, sialic acid, lactose, mannose, and GlcNAc) and the glycomic features 715 of Zika virus protein E that were observed through MALDITOF-MS. 


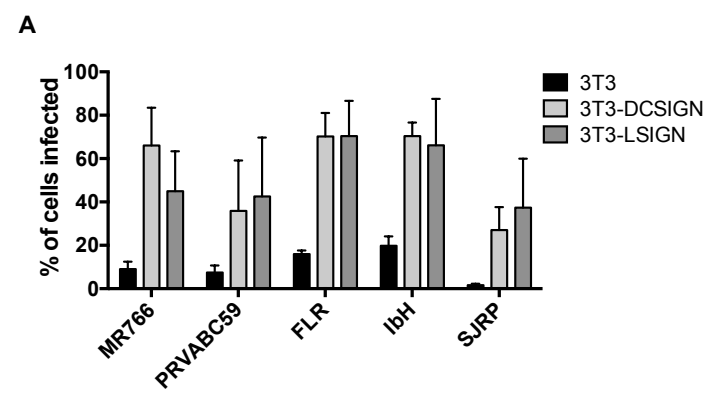

B

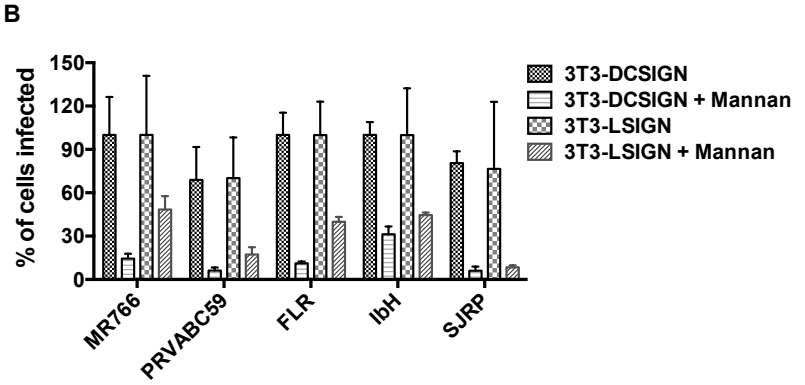

C

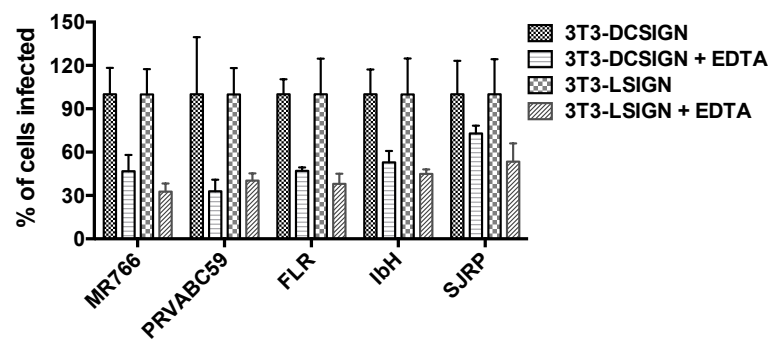

732

733

734

735

736

737

738

739

740

741

742

743

744

745

746

747

748

749

750
Fig. 5. The functional importance of $\mathbf{N}$-linked glycans on Zika virion infectivity. (a) DCSIGN/L-SIGN-mediated enhancement of Zika virus infection. 3T3, 3T3-DC-SIGN and 3T3-LSIGN cells were infected with ZIKV at MOI of 1, and the expression of viral E glycoprotein was detected 24 hours later through the use of an immunofluorescence assay. (b) DC-SIGN/L-SIGNmediated enhancement of Zika virus infection is inhibited by Mannan. 3T3-DC-SIGN or 3T3-LSIGN cell lines were incubated with Mannan (at $200 \mu \mathrm{g} / \mathrm{ml}$ ) inhibitors for $30 \mathrm{~min}$ at $37{ }^{\circ} \mathrm{C}$ and then infected with ZIKV at MOI of 1 . The same concentration was maintained constant throughout duration of infection. (c) DC-SIGN/L-SIGN-mediated Zika virus infection is reduced by EDTA. 3T3-DC-SIGN or 3T3-L-SIGN cell lines were preincubated with EDTA (at $0.5 \mathrm{mM}$ ) for $30 \mathrm{~min}$ at $37^{\circ} \mathrm{C}$ and then infected with ZIKV at MOI of 1 . The treatment was continued for 2 $\mathrm{h}$. After $2 \mathrm{~h}$, the virus and the EDTA containing medium was removed and continued the infection with the fresh medium. After $24 \mathrm{~h}$, the infected cells were detected using viral E protein antibody and the percent of infected cells was assessed by an immunofluorescence assay using Operetta High-Content Imaging System. Error bars indicate standard deviations calculated from 4 replicate wells in a 96-well plate. 